\title{
È possibile evidenziare le evidenze? Storia e fortuna di un calco anglicizzante
}

Riccardo Gualdo

PUBBLICATO: 30 GIUGNO 2020

\section{Quesito:}

Già da qualche tempo, e poi ancora in queste ultime settimane, che hanno portato alla ribalta tanti scienziati impegnati in Italia e all'estero nella lotta contro il contagio, arrivano domande a proposito dell'uso di evidenza, evidenze nel significato di 'prova, prove'; per esempio in evidenze scientifiche. È un uso d'origine inglese? È corretto in italiano?

\section{È possibile evidenziare le evidenze? Storia e fortuna di un calco anglicizzante}

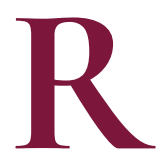

ispondere non è semplicissimo.

Evidenza è - evidentemente - un latinismo (tratto da evidens, participio di evidèri, del latino tardo), anticamente scritto anche evidenzia; una parola nata nel lessico colto, non certo nella lingua di tutti i giorni. Il suo significato principale è 'certezza, oggettività', tant'è vero che il nostro maggior dizionario storico, il GDLI, scrive cosi: "caratteristica per la quale una nozione, una perce. zione o una sensazione si presentano alla mente fornite di un cosi manifesto valore di verità da indurla all'assenso senza bisogno di prove o dimostrazioni" (corsivo di chi scrive); dunque, apparentemente, l'opposto di 'prova'. È lo stesso dizionario, però, a informarci che già Dante usò ad evidenza nel Convivio, intendendo appunto 'a dimostrazione, come prova'. Quest'accezione, tuttavia, restò marginale nella nostra lingua, limitata al lessico filosofico e religioso. Il fondatore dell'italiano scientifico moderno, Galileo, usa qualche volta evidenza, sempre al singolare e solo per inficiare la certezza fondata sull'esperienza dei sensi (evidenza matematica, geometrica evidenza; sono significative anche le combinazioni dell'aggettivo evidente: contrasti (cioè 'ragioni contrarie') evidenti e sensati, evidentissime prove, evidentissime esperienze); ma per il significato che c'interessa si serve quasi esclusivamente di prova e della famiglia di parole che ne deriva. Proprio l'origine latina di evidenza dà ragione della sua larga diffusione come europeismo del lessico intellettuale, e del fatto che in inglese almeno dal XVII secolo - si sia affermata l'accezione secondaria di 'prova', anche intesa come 'prova testimoniale' nel linguaggio giuridico.

Tornando all'oggi, nella lingua comune con evidenza indichiamo senza alcun dubbio, usando quasi sempre la forma singolare, quel che appare in modo chiaro e inequivocabile ai nostri sensi: l'evidenza di un fatto, di un fenomeno, arrendersi all'evidenza o negare l'evidenza, evidenza chiara, limpida, solare; analogo il valore di evidenza nella locuzione avverbiale con (ogni, tutta) evidenza. Secondario, ma pure frequente nell'uso, è il significato di 'risalto, rilievo' in frasi come dare evidenza (a qualcosa), (mettere, porre) in evidenza, su cui tornerò più oltre.

Il significato di 'prova' s'insinua con più insistenza nella lingua solo negli ultimi decenni del Novecento, e va di pari passo con l'aumento di frequenza della forma plurale e del costrutto "c'è (o non c'e) evidenza" "ci sono evidenze", prima non comune; lo conferma, nel motore di ricerca Google NGramViewer, la curva della combinazione evidenze scientifiche, confrontata con quella di prove scientifiche, che mi è stata segnalata dal professor Gianfranco Porcelli, già ordinario di linguistica inglese all'università di Pavia. Copio qui di seguito l'istantanea della ricerca: 


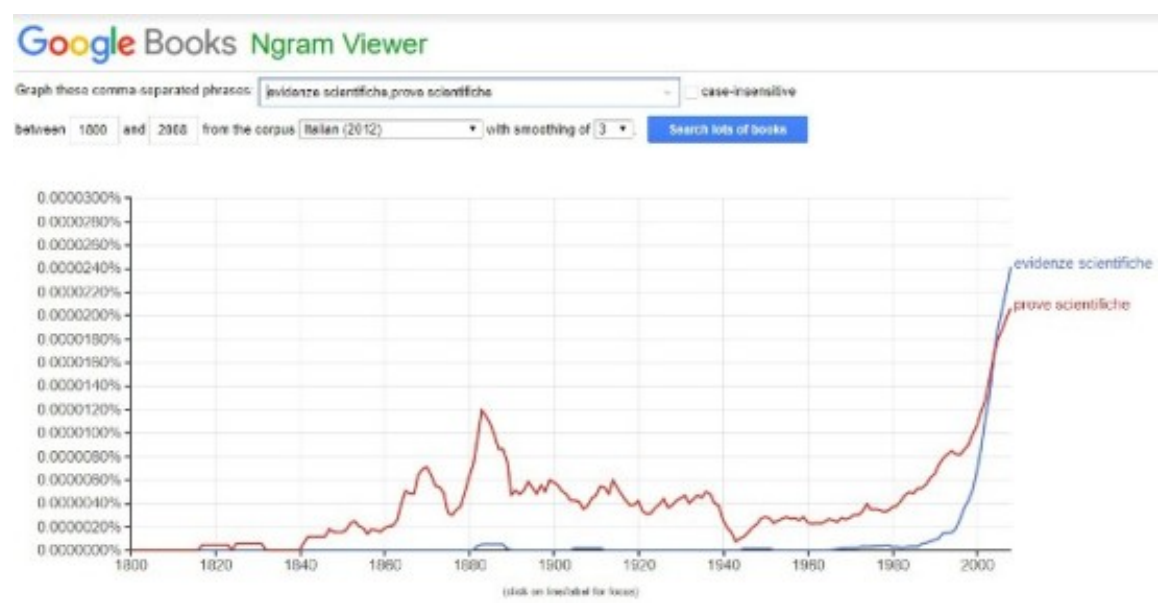

La linea blu supera d'un balzo quella rossa proprio alla fine del millennio. Dunque l'evidenza filosofica di Dante o di Giordano Bruno non c'entra: è una novità recente, che si deve senz'altro al modello dell'inglese.

Siamo di fronte a un calco semantico: un'accezione di una parola che mancava del tutto, oppure era rara, secondaria, si afferma e diventa via via più frequente per influsso di una parola straniera che ha medesima origine e simile aspetto formale. Un esempio di calco molto citato è quello del verbo realizzare, che al significato tradizionale di 'tradurre in realtà, attuare' ha aggiunto nel corso del Novecento quello di 'rendersi conto (di qualcosa), comprendere' per influsso dei significati che ha in inglese il verbo (to) realize. Cosi evidenza scientifica ricalca l'inglese scientific evidence 'prova scientifica'.

Il calco, però, non è solo semantico, ma tocca anche la sintassi e la morfologia, producendo in quest'ultima un'interessante diffrazione. Comincio dalla sintassi: nel suo significato finora prevalente, evidenza indica una qualità: l'evidenza di un fatto, di una dimostrazione, è la qualità di essere evidenti. Nella nuova accezione, l'evidenza è un fatto; di qui la possibilità dell'uso predicativo "c'é / non c'é evidenza scientifica di qualcosa", laddove prima avremmo detto "qualcosa è scientificamente evidente". La curiosità morfologica è la possibilità dell'uso plurale, assente nel modello; infatti in inglese evidence per 'prova' è solo singolare, come information e altri nomi astratti; in italiano era, di fatto, prevalentemente singolare, ma l'estensione del significato, che si spinge fino a sovrapporsi alla parola prova, la dispone a un uso al plurale che prima era poco frequente.

Può essere utile fornire qualche controprova.

Le prime riguardano la storia remota. Se cerchiamo la forma plurale evidenze in tutto il testo della versione elettronica del GDLI, da pochi mesi consultabile negli "Scaffali digitali" del portale dell'Accademia della Crusca, troviamo relativamente poco; alcune citazioni sono interessanti, come per esempio due luoghi della "Frusta letteraria" di Giuseppe Baretti, letterato e polemista piemontese del Settecento che visse a lungo, e mori, in Inghilterra, dove scrisse tra l'altro un fortunato dizionario bilingue inglese-italiano. Altri isolati esempi si ricavano da opere di Carlo Emilio Gadda e Tommaso Landolfi, celebri per il loro vocabolario ricercato e distante dalla lingua di tutti i giorni. Mi sembra la dimostrazione di un uso occasionale, che forse per Baretti risentiva già di qualche contatto con l'evidence inglese.

Un'altra controprova la traggo dalla seconda accezione di evidenza nell'italiano attuale. Anche nel significato di 'risalto, rilievo' evidenza indica un fatto, per quanto astratto; e tuttavia anche in quest'uso evidenza è rigorosamente solo singolare; nessun italiano madrelingua direbbe dare evidenze, mettere in evidenze. Di più, la parola è ancorata a combinazioni fisse con un numero limitato di verbi, 
tecnicamente verbi supporto, che consentono a un nome astratto - di solito un nome d'azione - di funzionare come se fosse un verbo; un verbo che esiste, in effetti: evidenziare.

All'orecchio dei puristi di fine Ottocento la locuzione mettere in evidenza suonava come un riprovevole francesismo; chissà che cosa avrebbero pensato di evidenziare, che dal GDLI è registrato, anche nel participio presente evidenziante e nel sostantivo evidenziatrice, solo con esempi tratti dall'opera di Gadda (il pennarello evidenziatore arriva un po' più tardi, e compare solo nei Supplementi del GDLI). I contesti gaddiani citati sono quasi sempre d'ambito medico; meglio, della consapevole parodia di un linguaggio tra il medico e il burocratico. Sono tra i primi segni di vitalita di un verbo oggi usatissimo, che ai puristi era sfuggito; devo l'informazione a Emiliano Picchiorri, che ha controllato nei repertori puristici otto-novecenteschi.

Evidenziare era sfuggito alla censura puristica perché non era ancora nato? Forse no. Ancora a Picchiorri devo il recupero - tramite la funzione "ricerca libri" di Google - di una precoce apparizione di evidenziare nel volume 25 del "Giornale di farmacia chimica e scienze accessorie". Siamo nel I847, e si sta parlando di antidoti all'arsenico. Il testo è un'esemplare miscela di lingua degli uffici (nel caso specifico, dell'amministrazione austriaca nel Lombardo-Veneto) e dei referti tecnici, qui farmaceutici (neretto mio):

Ora mi resta a far comprendere quanto sia necessitevole che i chimici-farmacisti esercenti, adempiendo doverosamente alle ingiunzioni della Superiorità, e i nostri all'ordinanza dell'eccelso Governo emessa con circolare delegatizia 17 giugno I847, si tengano muniti dellidrato umido o gelatinoso di ferro interamente alla condizione di sovrossido, escludendo quello i cui caratteri fisici e chimici evidenziano contenere frammisto l'ossido ferroso che non è contravveleno dell'arsenico.

Lo scrivente usa evidenziare con tranquillità, segno che non lo avverte come nuovo; possiamo dunque immaginare che circolasse già da qualche tempo.

La digressione è stata un po' lunga, ma spero non priva d'interesse.

Concludendo: evidenza ed evidenze per 'prova, prove' sono senz'altro un calco semantico sullinglese della comunità scientifica internazionale, che s'irradia in modo inconsapevole nell'italiano dei ricercatori: "non c'è alcuna evidenza - dichiarava il 9 maggio 2020 l'epidemiologo Andrea Crisanti in un'intervista televisiva - che l'aver contratto il CoVID-rg e poi esserne guariti renda immuni". Sarebbe probabilmente più chiaro dire prove, magari specificando prove sperimentali, cioè fondate su una rigorosa sperimentazione scientifica. L'autorevolezza degli scienziati e i contesti specialistici in cui circola rendono il modello prestigioso e imitabile: piuttosto che rimproverare chi dice evidenze sarebbe opportuno dedicare maggiore attenzione alle traduzioni, soprattutto a quelle specialistiche, nelle quali il rischio del calco è sempre dietro l'angolo.

Credo sia utile segnalare il travaso incipiente di evidenza 'prova' dalla lingua delle scienze (soprattutto le scienze della vita) a quella delle indagini forensi; dal sito RecoveryItalia.it ricavo la frase seguente: "Linformatica forense è un ramo della scienza forense applicata all'analisi di evidenze legali trovate in computer e periferiche di archiviazione di massa" (corsivo mio); e ancora, ecco come comincia, nel sito MasterLex.it, un articolo che descrive un programma informatico capace - a quanto pare - di simulare la sentenza di un giudice: "Utilizzare l'intelligenza artificiale per dirimere anche le questioni legali. È quello che hanno provato a fare un gruppo di computer scientist della University College London, mettendo a punto un software che fosse in grado di confrontare evidenze legali, principi e norme giuridiche, in modo da arrivare a una previsione quanto piu corretta possibile dell'esito della sentenza" (corsivo mio). 
Per ora l'espressione evidenze legali non sembra aver ancora sostituito prove legali, ma chissà che cosa succederà in un prossimo futuro.

\section{Cita come:}

Riccardo Gualdo, Ė possibile evidenziare le evidenze? Storia e fortuna di un calco anglicizzante , "Italiano digitale", 2020, XIII, 2020/2 (aprile-giugno)

DOI: $10.35948 / 2532-9006 / 2020.4347$

Copyright 2020 Accademia della Crusca

Pubblicato con licenza creative commons CC BY-NC-ND 